\title{
Effect of inlet turbulence on compressible flow through the tip-section turbine blade cascade with supersonic inlet
}

\author{
Petr Straka ${ }^{1, *}$, Jaromír Př́hoda ${ }^{2}$, David Fenderl $^{3}$, and Bartoloměj Rudas ${ }^{3}$ \\ ${ }^{1}$ VZLU Czech Aerospace Centre, Plc, Prague, Czech Republic \\ ${ }^{2}$ Czech Academy of Sciences, Institute of Thermomechanics, Prague, Czech Republic \\ ${ }^{3}$ Doosan Škoda Power Co., Ltd., Pilsen, Czech Republic
}

\begin{abstract}
The contribution deals with the numerical simulation of 2D compressible flow though the tip-section turbine blade cascade with the supersonic inlet boundary conditions. The simulation was carried out by the in-house numerical code using the explicit algebraic Reynolds stress model completed by the bypass transition model with the algebraic equation for the intermittency coefficient. The $\gamma$-Re model implemented in the commercial code Fluent was used for the comparison. Predictions carried out for the nominal conditions were focused on the effect of inlet free-stream turbulence on the flow structure in the blade cascade under supersonic inlet conditions. Numerical results were compared with experimental data.
\end{abstract}

\section{Introduction}

Flows in turbine blade cascades are influenced by many parameters which should be considered in numerical simulations. Such parameters are particularly the effect of freestream turbulence and possibly wall roughness that influence noticeably the transition to turbulence and the turbulent heat transfer. Moreover, the transonic and supersonic flows though the turbine blade cascade are considerably influenced by the interaction of shock waves with the boundary layer on the blade surface that can cause the transition to turbulence in separated flow. The character of the interaction depends not only on the shock wave intensity but also in large measure on the type of the boundary layer. At the interaction of the shock wave with the laminar boundary layer, the flow separation usually comes out followed by the transition in separated flow, while the turbulent boundary layer remains attached. Therefore, simulations were focused mainly on the effect of the inlet freestream turbulence on the flow structure in the blade cascade.

The contribution deals with the numerical simulation of compressible flow through the linear turbine blade cascade with blade profiles corresponding to the tip-section of the lowpressure part of the large steam turbine. Numerical simulations were carried out for the nominal regime partly by the in-house numerical code based on the EARSM turbulence

*Corresponding author: straka@,vzlu.cz 
model with the algebraic bypass transition model proposed by Straka and Příhoda [1] and partly by the $\gamma$-Re transition model connected with the SST turbulence model according to Langtry and Menter [2] implemented in the commercial code ANSYS Fluent.

\section{Mathematical model}

The mathematical model of compressible flow is based on conditionally-averaged NavierStokes equations completed by constitutional relations for the ideal gas, the explicit algebraic turbulence model (EARSM) by Hellsten [3], the algebraic transition model proposed by Straka and Př́hoda [1] and by the model of turbulent heat transfer based on the generalized gradient hypothesis according to Launder [4]. The EARSM model is modified into the form corresponding to models with the turbulent viscosity. The turbulent stress is given by the relation

$$
\tau_{i j}=\mu_{t}\left(2 S_{i j}-\frac{2}{3} \delta_{i j} \frac{\partial \bar{U}_{k}}{\partial x_{k}}\right)-\frac{2}{3} \delta_{i j} \rho k-a_{i j}^{(e x)} \rho k
$$

where the extra-anisotropy tensor $a_{i j}(e x)$ is dependent on strain-rate and vorticity tensors and their invariants. Turbulent scales are determined by the $k-\omega / k-\varepsilon$ turbulence model of Menter [5]. Turbulent time scale $\tau \sim 1 / \omega$ is replaced near the wall by the Kolmogorov viscous time scale. The production term in the turbulent energy equation is modified for the reduction of the over-production of the turbulent energy in the stagnation region. Production and destruction terms in the $k$-equation are multiplied by the intermittency coefficient $\gamma$. Similarly, the effective viscosity is given by $\mu_{e f}=\mu+\gamma \mu_{t}$ in the transition region. The EARSM model including the prescription of boundary conditions is described by Hellsten [3]. Some modifications of the model are given by Straka and Prríhoda [6].

The generalized gradient hypothesis is used for modelling of the turbulent heat transfer in the form

$$
q_{i}=-C_{t} \frac{\tau_{i j}}{\omega} \frac{\partial \bar{T}}{\partial x_{j}} \quad C_{t}=0.3
$$

The transition model is based on the concept of different values of the intermittency coefficient in the boundary layer $\gamma_{i}$ and in the free stream $\gamma_{e}$. The intermittency coefficient in the boundary layer $\gamma_{i}$ is expressed by the relation

$$
\gamma_{i}=1-\exp \left[-\hat{n} \sigma\left(R e_{x}-R e_{x t}\right)^{2}\right]
$$

The transition onset is given by the empirical correlation for the momentum Reynolds number

$$
R e_{\theta t}=R e_{\theta t o}\left[1+\mathrm{F}(T u) \frac{1-\exp (-40 \lambda)}{1+0.4 \exp (-40 \lambda)}\right]
$$

where the transition onset on the flat plate without the pressure gradient is given by the relations

$$
\begin{array}{rc}
R e_{\theta t o}=975.8-497.2 T u+\frac{11.4}{T u} & \text { for } \quad T u \leq 1 \% \\
\operatorname{Re}_{\theta t o}=96.7+\frac{340}{T u}+\frac{53.3}{T u^{2}} & \text { for } \quad T u>1 \%
\end{array}
$$

depending on the free-stream turbulence level $T u(\%)$ and the pressure-gradient parameter $\lambda_{t}$. The length of the transition region is expressed using the parameter

$$
N=\hat{n} \sigma \operatorname{Re} \theta_{t}^{3}
$$


combining the spot generation rate $\hat{n}$ and the spot propagation rate $\sigma$ introduced by Narasimha [7]. The parameter $N$ for the attached flow is given by the empirical relation $N=$ $\mathrm{f}\left(\mathrm{Tu}, \lambda_{t}\right)$ proposed by Solomon et al. [8]. The onset of transition in separated flow is given by the correlation according to Mayle [9] in the form

$$
R e_{x t}=300 R e_{\theta s}^{0.7}+R e_{x s}
$$

where $\operatorname{Re} \theta_{s}$ is the momentum Reynolds number at the separation and $R e_{x s}$ is the Reynolds number related to the distance of the separation from the leading edge. The transition length of the short separation bubble is given according to Walker [10] by the relation

$$
\hat{n} \sigma=\frac{R e_{x t}^{-1.34}}{40}
$$

and so the same approach can be applied as in the attached flow.

The application of local variables is necessary for prediction of transitional flows in complex geometries. Therefore, the momentum Reynolds number is replaced by the maximum of the vorticity Reynolds number according to Langtry and Menter [3]. The vorticity Reynolds number is given by the relation

$$
\operatorname{Re}_{\Omega}=y^{2}|\Omega| / v
$$

where $y$ is the distance from the wall and $\Omega$ is the absolute value of the vorticity tensor. The link between both Reynolds numbers is expressed by the relation $R e_{\theta}=R e_{\Omega \max } / C$ where the parameter $C$ depends on the pressure gradient expressed by the parameter

$$
L=R e_{\Omega}^{2} \frac{v}{U_{e}^{2}} \frac{\mathrm{d} U_{e}}{\mathrm{~d} x}
$$

The variation of the parameter $C$ with the pressure gradient parameter $L$ was estimated by means of similar solutions of Falkner-Skan velocity profiles.

The system of governing equations was implemented into the in-house numerical code based on the finite volume method of the cell-centered type. The governing equations are discretized using a multi-block quadrilateral structured grid with a block overlapping implementation. The algebraic transition model is described in detail by Fürst et al. [11].

The correlation-based $\gamma-\operatorname{Re}_{\theta}$ model of the bypass transition proposed by Langtry and Menter [3] with the two-equation SST turbulence model implemented into the commercial code ANSYS Fluent was used for comparison. The transition model is based on transport equations for the intermittency coefficient and the transitional momentum Reynolds number. The intermittency coefficient $\gamma$ is given by the transport equation

$$
\frac{\partial(\rho \gamma)}{\partial t}+\frac{\partial\left(\rho U_{j} \gamma\right)}{\partial x_{j}}=P_{\gamma}-E_{\gamma}+\frac{\partial}{\partial x_{j}}\left[\left(\mu+\frac{\mu_{t}}{\sigma_{\gamma}}\right) \frac{\partial \gamma}{\partial x_{j}}\right]
$$

where

$$
P_{\gamma}=F_{\text {length }} \mathrm{C}_{a 1} \rho S\left(\gamma F_{\text {onset }}\right)^{1 / 2}\left(1-\mathrm{C}_{e 1} \gamma\right)
$$

is the production term with empirical constants $F_{\text {onset }}$ for the transition onset and $F_{\text {length }}$ for the transition length. The application of local variables is allowed by the relation between the Reynolds number related to the momentum thickness and the maximum of the vorticity Reynolds number used in the form $R e_{\theta}=R e_{v_{\max }} / 2.193$ valid for the Blasius boundary layer. The empirical correlation for the transition onset $F_{\text {onset }}$ depends on the critical Reynolds number $R e \theta_{\mathrm{c}}$ giving the position where the turbulent energy begins to grow up, see Langtry [12]. This Reynolds number $\operatorname{Re}_{\theta_{c}}$ is expressed by means of the local Reynolds number $\tilde{R} e_{\theta t}$ for the transition onset determined from the transport equation 


$$
\frac{\partial\left(\rho \tilde{R} e_{\theta t}\right)}{\partial t}+\frac{\partial\left(\rho U_{j} \tilde{R} e_{\theta t}\right)}{\partial x_{j}}=P_{\theta t}+\frac{\partial}{\partial x_{j}}\left[\sigma_{\theta t}\left(\mu+\mu_{t}\right) \frac{\partial \tilde{R} e_{\theta t}}{\partial x_{j}}\right]
$$

The onset and length of the transition region, i.e. parameters $R e_{\theta_{c}}$ and $L_{\text {length }}$ are expressed by means of the local Reynolds number $\tilde{R} e_{\theta t}$. The model is switched over to a simple algebraic transition model in the case of the transition in separated flow. The onset and the length of the transition region are controlled by threshold functions based on empirical correlations. The transition model is based on local variables only and can be used for the simulation of complex flows using unstructured computational grids. The $\gamma-R_{\theta}$ model with all necessary empirical correlations was published by Langtry and Menter [3].

\section{Results}

Numerical simulations of two-dimensional compressible flow through the linear tip-section turbine blade cascade TR-U-6 were carried out for practically nominal conditions, i.e. for the inlet Mach number $M_{l}=1.68$ and the isentropic outlet Mach number $M_{2 i s S C}=2.05$ corresponding to the pressure in the settling chamber. The relevant isentropic outlet Mach number in the traversing plane is $M_{2 i s}=1.97$ and the isentropic outlet Reynolds number $R e_{2 i s}=1.98 \times 10^{6}$. Numerical results were compared with results of optical and pressure measurements, see Luxa et al. [13, 14].

The scheme of the computational domain for simulations by the EARSM turbulence model with the algebraic transition model is shown in Figure 1. The detail of the computational mesh of the "chimera" type near the leading and the trailing edge is given in Figure 2. The mesh consists of the O-type block around the blade profile and the H-type block which covers one pitch of blade cascade. The computational grid is refined near the wall with the first grid node in the distance from the wall about $y^{+}=1$.

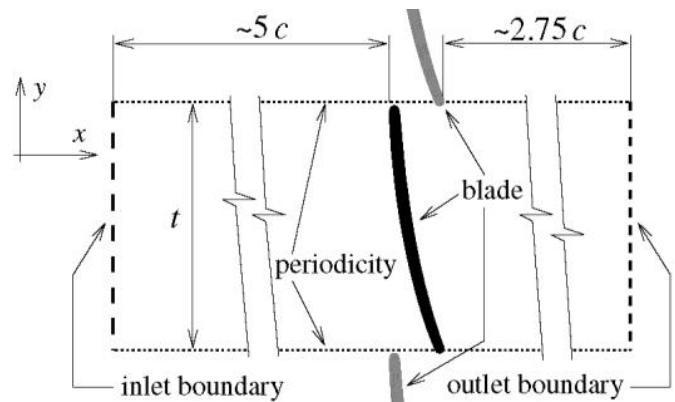

Fig. 1. Scheme of the computational domain.

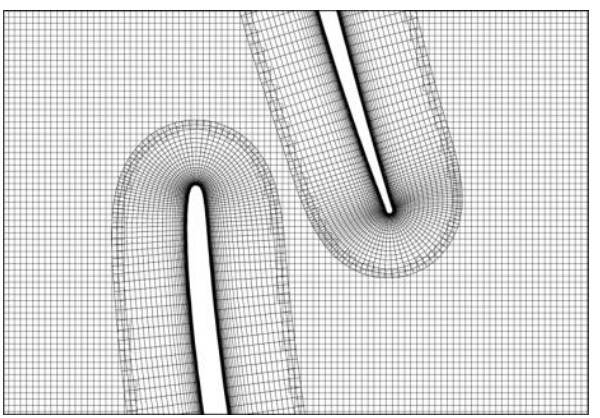

Fig. 2. Detail of computational mesh.

Because of supersonic inlet conditions the flow through the blade cascade is influenced by parasitic shock waves arising by the reflection from boundaries of the domain. The application of the quadrilateral block-structured computational grids with their overlapping leads moreover to the reflection of shock waves on the block boundaries. Therefore the domain was extended in $x$-direction upstream and downstream to suppress these reflections. The constant values of the total pressure $p_{01}$, total temperature $T_{01}$ and incidence angle $\alpha_{l}$ were prescribed as inlet conditions. The static pressure was extrapolated from the computational domain. The static pressure given by the outlet isentropic Mach number was prescribed as the outlet condition. Periodicity conditions were applied on corresponding free side boundaries of the computation domain. The inlet turbulence parameters, i.e. turbulence level $T u$ and the ratio of turbulent and molecular viscosity $\mu_{t} / \mu$ are chosen due to the turbulence decay according to the length of the inlet part. The relevant 
values of the turbulence level in the distance about one spacing upstream of the plane of the leading edges were $T u=1,1.5$ and $2.5 \%$ where the value $T u=1.5 \%$ corresponds to experimental data.

The computational domain used in calculations by means of the commercial code ANSYS Fluent is shown in Figure 3. The domain was extended before and behind the blade cascade to $3 c$. The mesh was formed by quadrilateral elements refined near walls and by triangular elements in the extended parts of the domain to suppress the effect of shock waves reflections. The detail of the grid near the blade is shown in Figure 4. The same inlet conditions were used as for the numerical simulation by means of the in-house code.

Due to supersonic inlet the prescription of inlet boundary conditions is rather complicated. The flow angle is prescribed at the inlet boundary in numerical simulations. The relation between values of the inlet Mach number and the inlet angle is given by the unique incidence rule (see e.g. Luxa et al. [15]). The relevant inlet conditions were established in the distance $50 \mathrm{~mm}$ upstream of the leading edge plane.

The data reduction method proposed by Amecke and Šafaŕík [16] was used for the evaluation of experimental data and results of CFD simulations as well. Due to the stagger angle of blades and to the inlet boundary conditions the concave side of the blade is the suction side and the convex side is the pressure side.

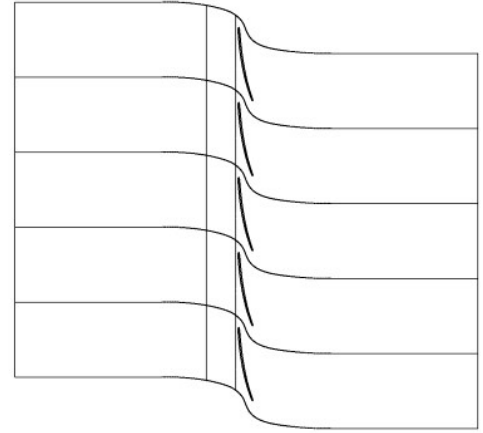

Fig. 3. Scheme of the computational domain (Fluent).

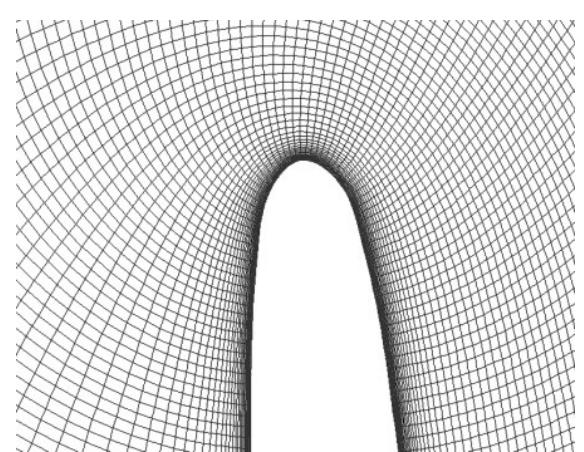

Fig. 4. Detail of computational mesh(Fluent).

Simulations carried out for the nominal regime were focused mainly on the effect of the inlet free-stream turbulence on the flow field in the blade cascade especially on the interaction of the impinging shock wave with the boundary layer on the suction side. Simulations are to-follow up to predictions carried out by Straka et al. [17] for different flow conditions where the effect of the inlet angle and the free-stream turbulence was studied.

The flow field in the tip-section blade cascade is influenced partly by the interaction of internal branch of exit shock wave with boundary layer on the suction side of neighbouring blade and partly by the interaction of internal branch of inlet shock wave upstream the blade leading edge with boundary layer on pressure side of previous profile. At higher inlet Mach number this inlet shock wave is to that extent curved that it pasts the suction side of the previous blade and propagates downstream, see Luxa et al. [18].

The interferometric and schlieren pictures obtained for nominal conditions, i.e. for the inlet Mach number $M_{l}=1.68$ and the isentropic outlet Mach number for $M_{2 i s}=1.97$ are shown in Figure 5 and 6 . Due to a relative high inlet Mach number $M_{l}$ only the interaction of internal branch of exit shock wave with boundary layer on the suction side of neighbouring blade occurs. This interaction with the laminar boundary layer leads to flow separation. 


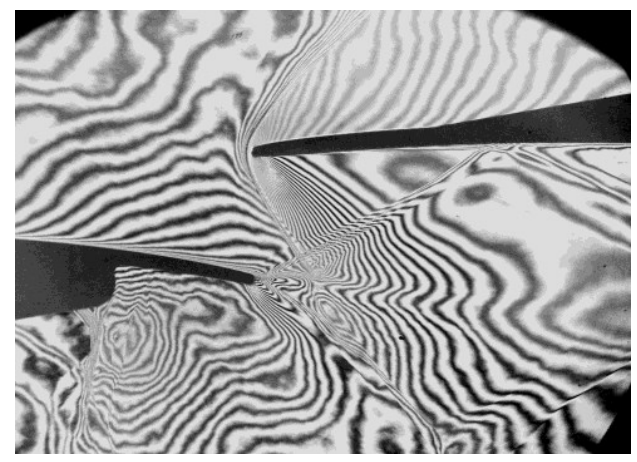

Fig. 5. Interferometric picture for $M_{2 i s}=1.97$.

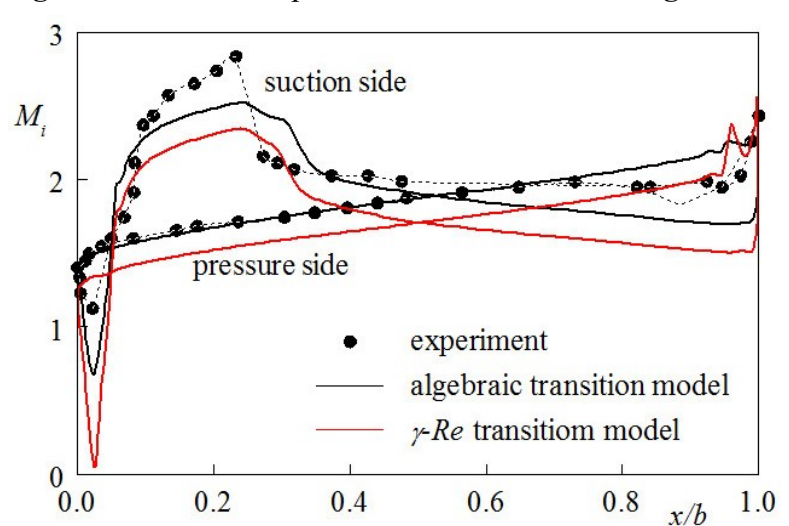

Fig. 7. Distribution of the isentropic Mach number.

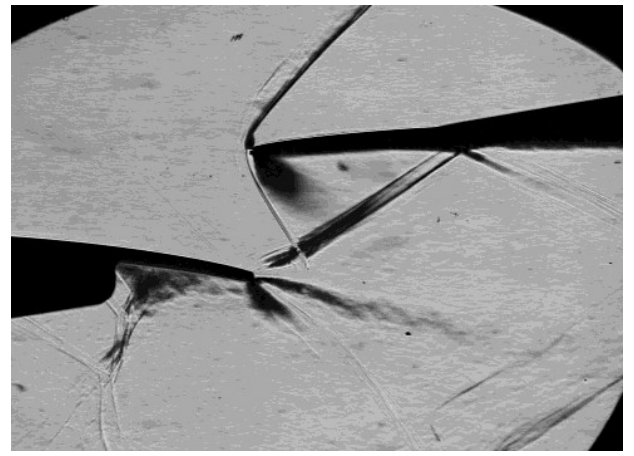

Fig. 6. Schlieren picture for $M_{2 i s}=1.97$.

The distribution of the isentropic Mach number along the blade for $T u \approx 1.5 \%$ is compared with experimental data in Figure 7. The distance $b$ represents the axial chord length. The flow field in the interblade channel is supersonic with a small subsonic region around the leading edge. The EARSM model with the algebraic transition model quite well corresponds with experi-mental data except the part upstream of the interaction. The SST turbulence model with $\gamma$-Re transition model gives generally rather lower values $M_{i}$ with a similar form as the algebraic model. It should be caused by the used isentropic outlet Mach number $M_{2 i s}=1.94$ instead of the nominal value. Only small changes in the distribution of the isentropic Mach number occur with changes of turbulence on the suction side in the sudden decrease of the velocity. The Mach number isolines at $M_{2 i s}=1.97$ obtained by the algebraic transition model are shown in Figure 8 .

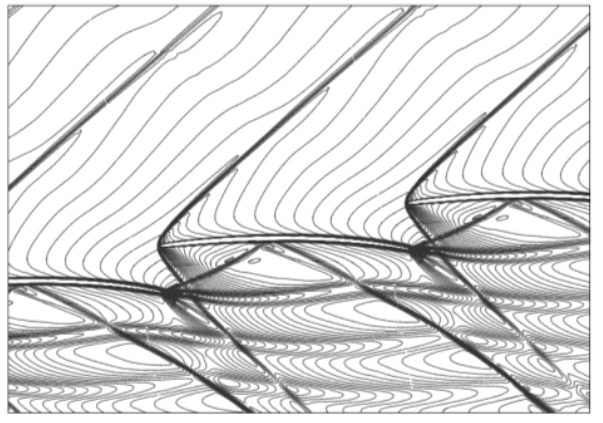

Fig. 8. Mach number isolines in the blade cascade.

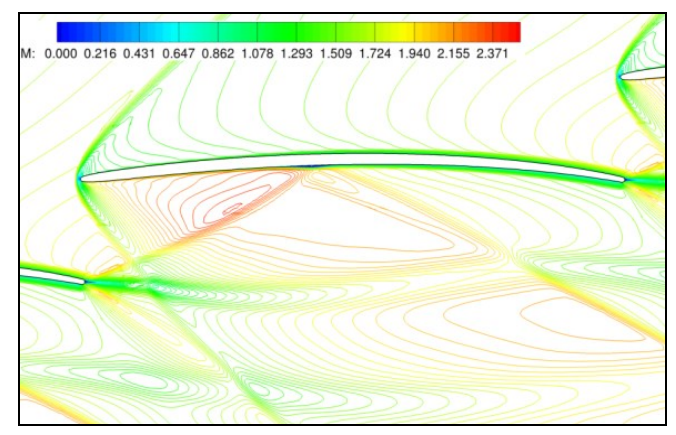

Fig. 9. Detail of Mach number isolines.

The detail of Mach number isolines showing the interaction of the inner branch of the exit shock wave with the boundary layer on the suction side of the blade is presented in Figure 9. The field of Mach isolines well corresponds with the interferometric picture. The impact of the shock wave and the interaction with the boundary layer induces flow separation connected with the transition to turbulence. The similar flow field structure was 
obtained as well by means of the $\gamma$-Re transition model as can be seen in Figure 10 . The flow structure in the blade cascade apparent in the field of the Mach isolines can be well documented by means of the distribution of the skin friction coefficient.

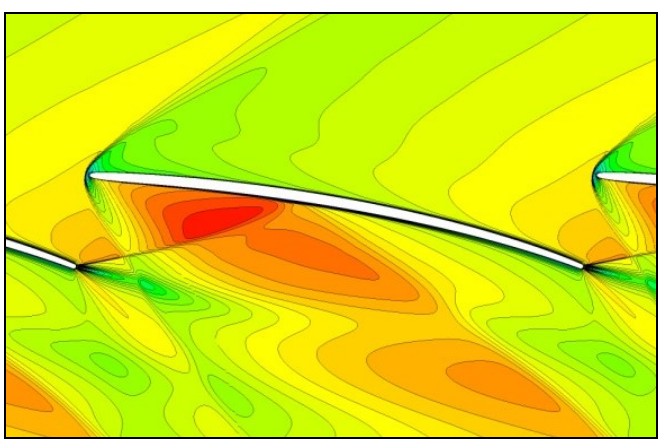

Fig. 10. Mach number isolines near blades (Fluent).

The skin friction coefficient obtained by the algebraic transition model for various free-stream turbulence levels is shown in Figure 11. The skin friction coefficient is defined by the relation $C_{f}=$ $\tau_{w} / p_{d l}$ where $p_{d l}$ is the inlet dynamic pressure. The extent of the separation zone estimated from experimental data is marked by the rectangle and is approximately from $x / b \approx 0.28$ to 0.40 . The transition onset on the pressure side moves upstream with the increasing freestream turbulence. A quite different situation is on the suction side where the transition is caused by the shock-wave interaction with the laminar boundary layer. This interaction has as a consequence the flow separation and the transition in separated flow. The extent of the separation zone becomes shorter with the increasing turbulence level.

The skin friction distribution obtained by both transition models for the free-stream turbulence about $T u \approx 1.5 \%$ are compared in Figure 12. Results for the suction side are very similar including the modelling of the separation zone. Nevertheless, the $\gamma$-Re model demonstrates some oscillations of the skin friction near the trailing edge. On the pressure side, the algebraic transition model predicts the transition onset near the leading edge at $x_{t} / b$ $\approx 0.03$ while $\gamma-R e$ model gives the transition onset about at $x_{t} / b \approx 0.18$ with a rather long transition region.
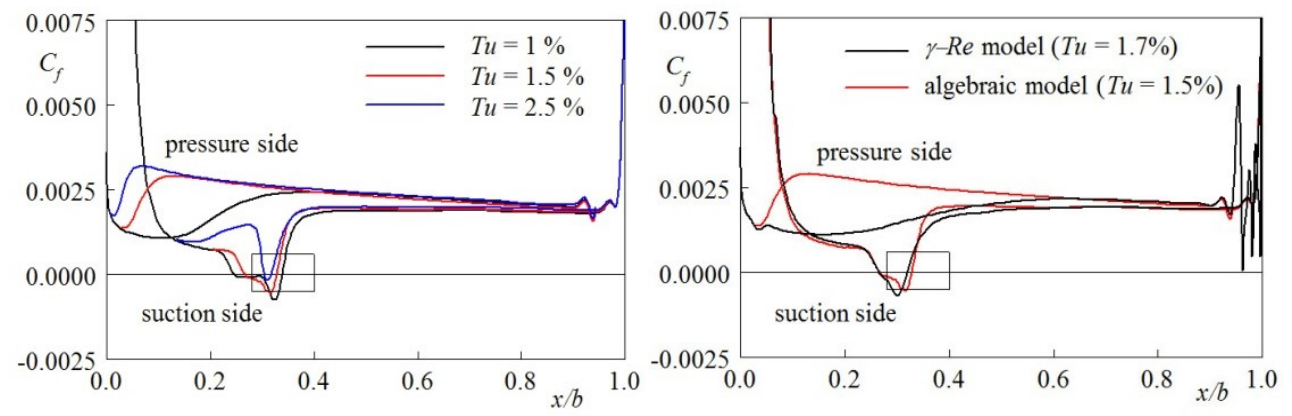

Fig. 11. Skin friction distribution distribution (algebraic model).

Fig. 12. Comparison of the skin friction for both transition models.

The field of the turbulent energy around the blade obtained by the EARSM turbulence model with the algebraic transition model is shown for various turbulence levels in Figures $13 \mathrm{a}$ ) and b). The transition in the separated flow for $T u=1.5 \%$ results in the greater growth of the turbulent energy on the suction side towards to the trailing edge while the transition zone for $T u=2.5 \%$ includes a very small separation and so the growth of the turbulent energy is rather smaller. On the contrary, the transition onset on the pressure side moves upstream with the increasing turbulence and so the length of turbulent boundary layer becomes longer which leads a higher turbulent energy. 


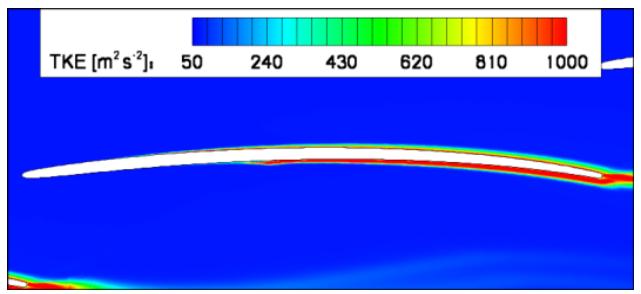

a) $T u=1.5 \%$

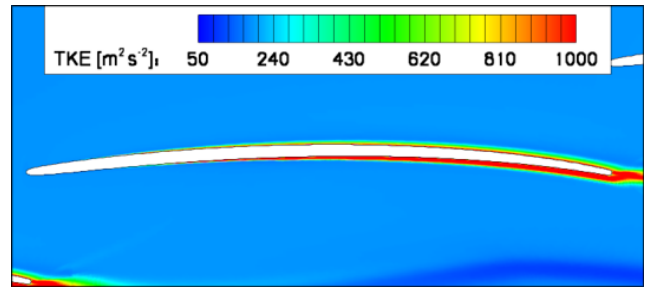

b) $T u=2.5 \%$

Fig. 13. Field of the turbulent energy around the blade (algebraic transition model).

\section{Conclusion}

The explicit algebraic model of Reynolds stresses with the algebraic transition model based on local variables was used for the numerical simulation of compressible flow through the tip-section turbine blade cascade TR-U-6 with supersonic inlet conditions. The calculations by the $\gamma$-Re transition model with the SST turbulence model implemented in the commercial software ANSYS Fluent was carried for the comparison. Simulations were focused especially on the effect of the inlet free-stream turbulence on the interaction of the exit shock wave with the boundary layer on the blade suction side. Obtained results indicate the significant effect of the transition modelling on the adequate description of the compressible flow through the blade cascade. The application of the advanced turbulence models with the adequate transition model for attached and separated flow is necessary.

The work was supported by the Technology Agency of the Czech Republic under the grant TA02020057 and by the Long-term Framework Advancement Plan provided by the Ministry of Industry and Trade of the Czech Republic. Institutional support RVO 61388998 is also gratefully acknowledged.

\section{References}

1. P. Straka, J. Př́ihoda, Proc. Conf. Experimental Fluid Mechanics, Liberec, 636-641 (2010)

2. R.B. Langtry, F.R. Menter, AIAA Jour., 47, 2894-2906 (2009)

3. A. Hellsten, New two-equation turbulence model for aerodynamics applications, $\mathrm{PhD}$. thesis, Helsinki University of Technology (2004)

4. B.E. Launder, Jour. of Heat Transfer, 110, 1112-1128 (1988)

5. F.R. Menter, AIAA Jour., 32, 1598-1605 (1994)

6. P. Straka, J. Př́ihoda, Proc. Conf. Topical Problems of Fluid Mechanics, Praha, 131134 (2014)

7. R. Narasimha, Jour. Aerospace Science, 24, 711-712 (1957)

8. W.J. Solomon, G.J. Walker, J.P. Gostelow, Jour. Turbomachinery, 118, 744-751 (1996)

9. R.E. Mayle, Jour. Turbomachinery, 113, 509-537 (1991)

10. G.J. Walker, AIAA Jour., 27, 595-607 (1989)

11. J. Fürst, J. Př́ihoda, P. Straka, Computing, 95, S163-S182 (2013)

12. R.B. Langtry, A correlation-based transition model using local variables for unstructured parallelized CFD codes, PhD. thesis, Univ. Stuttgart (2006) 
13. M. Luxa, D. Šimurda, J. Fořt, J Fürst, P. Šafařík, J. Synáč, B. Rudas, Proc. $11^{\text {th }}$ European Conf. on Turbo-machinery, Fluid Dynamics and Thermodynamics (ETC11), Madrid (2015)

14. M. Luxa, J. Př́íhoda, D. Šimurda, P. Straka, J. Synáč, Jour. Thermal Science, 25, 138144 (2016)

15. M. Luxa, B. Rudas, J. Synáč, P. Šafař́k, D. Šimurda, Proc. Conf. Application of Experimental and Numerical Methods in Fluid Mechanics and Energy 2014, 149-152, Žilina (2014)

16. J. Amecke, P. Šafařík, Date reduction of wake flow measurements with injection of an other gas, DLR-Forschungsbericht 95-32, Göttingen (1995)

17. P. Straka, J. Př́hoda, M. Bobšík, Proc. Conf. Topical Problems of Fluid Mechanics, Praha, 207-2014 (2016)

18. M. Luxa, D. Šimurda, J. Příhoda, J. Váchová, VII European Congress on Computational Methods in Applied Sciences and Engineering (ECCOMAS), Crete, $10 \mathrm{p}$. (2016) 